\title{
Monitoring the standards of care in the hospital setting*
}

\author{
S. J. Johnston, Lecturer in Mental Health (Mental Handicap), University of Bristol, \\ Bristol BS2 8DZ
}

The way in which doctors practise medically undoubtedly has effects on the ways in which other staff, the nurse, occupational therapist, physiotherapist etc. are able to practise, just as their style affects the doctor. But all share the common aim of providing the best possible care for their patients. Changing service provisions have affected mental handicap services more than most. It is crucial at a time of great change within health service systems that good medical practices evolved over many years experience are not lost or neglected by changing philosophies or care models. Medical aspects of care of the mentally handicapped person which evolved in hospitals should be flexible enough to be used in the settings used by any district. Medical standards of care must be maintained and improved despite changes to the service.

The evolution of clinical operational policies in the Stoke Park Group of Hospitals (Frenchay Health District, Bristol) is discussed in terms of the documentation of previous hospital practice.

Extended use of operational guidelines is presented and discussed.

\section{Hospital systems of operation}

The hospital systems, until now, have relied upon constant senior staff within a single treatment setting, that of the hospital ward. The strict hierarchical system meant that information of the ongoing running of the service was passed down from the senior permanent members of the team to those in the transient junior training grades. The constancy of the senior staff, both medical and nursing, and the repeated reinforcement of the service structure they were operating served two purposes:

(a) Negative: New ideas may have been difficult to incorporate, the policies governing the service seemingly entirely dependent on the desires of those longest in the service, and therefore difficult to challenge, or even to see and learn, for new recruits to the service.

(b) Positive: Practices which were of benefit to the patient and to the longer term running of the

* This paper is based on a presentation at the Section for the Psychiatry of Mental Handicap Meeting held on 30 March 1990 at the University of Bristol. service were maintained as common knowledge, with constant reinforcement. Little of this was written down as operational policies. The staff were constant, there were always senior staff around to coach new arrivals.

However, in mental handicap, perhaps more than in any other specialty so far, this internal structure has been eroded. Internal and external influences have changed the entire shape of the service provided, with gradual reduction of the hospital bases and expansion of the community service.

The consultant is no longer actively involved in all aspects of medical care of the mentally handicapped. A review of consultant responsibility noted that in the hospital setting, the consultant is still responsible for all of the medical care of their patients, short or long stay (Berney, 1989). But less of the consultant's time will be spent on the hospital site, thereby requiring junior staff to provide the ongoing health care. The consultant may only make very brief ward visits and discuss individual patients at length only at case conferences.

With the changing role of the consultant psychiatrist in mental handicap the emphasis in training has become more specialised and much of the socalled common knowledge of a more general medical nature is at risk of being lost.

Certainly in our hospital group, regular and full medical reassessment was performed at least annually to detect early signs of treatable physical illness or disease which may otherwise have further handicapped an individual. These were performed by the specialist staff previously. Those people who had conditions known to be associated with physical problems were screened particularly.

As senior staff have been replaced, and the clinical workload of the present senior staff seems to be increasing with the diverse and complex needs of an integrated total service, it has become apparent that some of the worthwhile elements of the medical model for the care of medical conditions occurring in those people with a mental handicap are being swamped by a few well intentioned but mis-directed staff.

Further, with increasingly stringent training requirements for junior staff, they are allowed far less service commitment, have increasing educational activities, with more rapidly rotating clinical attachments; this may result in a lesser commitment to the 
long term functioning of the service. The tendency is to provide a crisis intervention service rather than one of continuing care. One solution has been the employment of clinical assistants or general practice hospital practitioners to provide day to day medical care but it is well known that teaching of the range of medical conditions pertinent to care of the mentally handicapped is scarce. It may take years to acquire the required knowledge.

More recently, mental handicap hospital services have been troubled by shortages of staffing, especially trained nurses, large numbers of experienced staff taking up appointments in the community, and others leaving the service altogether. The hospital based junior may no longer have the support of an experienced nursing team.

\section{Clinical operational policies}

During a regular Care Team Meeting at Stoke Park Hospital, attention was drawn to the need for all the female patients to have cervical smears. However, no mention was made of breast screening. A review of the available data revealed that in $\mathbf{4 5}$ years there had been no deaths from cervical carcinoma (although 1 uterine $\mathrm{Ca}$ ) but 17 deaths were related to breast carcinoma. Although possibly not surprising to medical personnel aware of the respective populations at risk, it was certainly surprising to the Stoke Park Hospital multidisciplinary team.

As a result of this meeting a document outlining the principles of Preventive Health Care was prepared (App.A). (Available on request from author.) Individual clinical policy documents are now being compiled to supplement the theory with detail. Each policy statement discusses the evidence for, and benefits of, the screening procedure under consideration.

(a) Well established principles for treatment are stated; for example, in the treatment of epilepsy, monotherapy should be used whenever possible. The control of seizure activity should not be at the expense of well-being and loss of skills or cognitive ability.

(b) The population at risk or eligible for screening are identified. For example, for anticonvulsant therapy, all patients should be included regardless of the reason for therapy i.e. whether epilepsy, affective disorder or aggressive behaviour.

(c) The parameters to be screened, any conditions for the screening and the frequency of screening are outlined.

(d) Levels of intervention are determined; for example, cervical screening of women; unless there are clear clinical grounds to suspect pathology any known virgo intacta or very difficult to examine patients should not be referred for cervical smears or examination under general anaesthetic.

A policy for Well Woman screening has now been written and discussed and commented on by the gynaecologists and all the medical staff and is now in operation (App.B). (Available on request from author.)

Further policies are now being written to cover such issues as:

(a) use and monitoring of anticonvulsants

(b) use and monitoring of lithium

(c) monitoring patients on psychotropic medication

(d) medical review of the elderly at $60 / 65$

(e) special screening, e.g. Down's Syndrome

(f) the annual physical examination.

It is hoped that this format which is easily modifiable with new medical advances will preserve the good practices which have been developed during many years of clinical experience. By making them accessible and available to new staff they may in addition provide an educational tool which could be further developed for those in the community.

Further, by defining operational policies, the process of clinical audit is simplified, and measurable criteria have been defined. For example, having identified breast carcinoma as an easily detectable condition, one measure of the effectiveness of the annual physical examinations could be to check that all female patients had had their breasts examined. Another measure, given that cook/chill food, designed for acute hospital patients is provided for this hospital group, would be regular checks of weight or monitoring of iron and vitamin status. These may seem trivial matters but an article published in the BMJ last year discussed the under nourishment and electrolyte disturbances in patients in a mental handicap hospital in comparison to the local psychiatric hospital (Macdonald et al, 1989).

Charles Shaw from the King's Fund College, London, visited Frenchay Health District, to conduct a workshop on clinical audit and visited independently both the Southmead and Frenchay Health Districts to speak to clinicians. As a consequence of his visits the monthly academic meeting of consultants and senior registrars in mental handicap psychiatry was extended to undertake audit. The review comprises five health districts and almost 1.5 million people in Avon and Gloucestershire. It was agreed that the audit findings should remain clinical, and confidential.

An assessment of the numbers of anticonvulsant drugs being received by Frenchay in-patients is as follows.

The total number of patients currently in the Stoke Park Group of Hospitals is 578. There are 176 
patients receiving anticonvulsant medication i.e. $30.5 \%$, the greatest percentage being at Stoke Park where the most disabled patients are.

Use of anticonvulsants is as follows: (a) Carbamazepine

(b) Sodium Valproate

(c) Clobazam

(d) Acetazolamide

(e) Clonazapam

(f) Phenytoin

(g) Phenobarbitone

(h) Primidone

\section{2}

42

29

6

2

2

2

2
$80.7 \%$

$23.7 \%$

$16.5 \%$

$3.4 \%$

$1.1 \%$

$1.1 \%$

$1.1 \%$

$1.1 \%$
At the time of the original survey one patient was taking Vigabatrin which has since been withdrawn. There are a number of patients, 34 (19.3\%) taking more than one anticonvulsant. The patients who have Lennox-Gastaur epilepsy, or who are currently receiving transitional therapy, totalled $13(7.4 \%)$. of:

The remainder, $21(12 \%)$, were on combinations

\section{Carbamazepine/Sodium}

Valproate + Clobazam

Carbamazepine + Sodium Valproate

16

$9.1 \%$

Carbamazepine + Acetazolamide

Carbamazepine + Phenytoin + Primidone

It is believed that as a result of maintaining high staff awareness of epilepsy in these hospitals there is a modern drug profile and a small percentage of polypharmacy. It is to maintain these ideals that operational policies are produced. The epilepsy clinic model may yet be considered but it will need a great deal of time and resources in setting it up. In the meantime clear written guidelines can only help.

\section{Medical input to nurse training}

Another means by which to monitor and maintain the standards of care in the hospital setting is in nurse education. As already mentioned, there is medical input to a Hospital Care Team where policy issues can be discussed; but there is ample opportunity for continuing education for staff in the medical conditions affecting patients. This may be in the form of informal ward discussion, extended case conferences, class-room teaching of nurse students or in-service training seminar days. The medical staff in Frenchay all contribute usefully to the above. The success of the seminars is evident in that they are continuing, on a greater range of topics.
Notably, Dr L. Cooke (Stoke Park Hospital) has been active in training days dealing with the problems faced by people with sensory handicaps in addition to mental handicap. Dr Y. Wiley (Stoke Park Hospital) was recently involved in training days covering, among other things, the Mental Health Act 1983, Control Restraint and Seclusion policy. The lecturer has been involved in teaching sessions for day hospital staff on a range of topics, i.e. epilepsy, psychiatric illness in mental handicap and medical complications of specific syndromes. Both Dr Cooke and the lecturer will shortly be taking part in a series of in-service training days on psychiatric illness in mental handicap.

\section{Comments}

It is hoped that clinicians will be stimulated to set up ways of monitoring standards of care in the hospital setting. As the service continues to change shape there may be even less opportunity for contact between colleagues. If the patients' standard of medical care is not to fall, or be lost until it is re-invented, steps must be taken to ensure that common knowledge is available to those who get it when they need it.

It is suggested that, in the absence of the tight hospital structure with regular morning meeting of medical staff and high degree of supervision of patient care directly by consultants, the provisions of flexible but clear clinical guidelines for the regular monitoring of important conditions or therapies is essential. These will determine the minimum standard of clinical supervision desired, form a basis for clinical audit, and allow clinical research into the efficacy of differing forms of treatment. Further, to ensure the support of other staff in adequate monitoring and treatment of medical conditions they must receive education on such conditions.

Finally, no small group of practitioners can set up systems of operation without being prepared to share and discuss the failures as well as the successes, and invite comment or inspection from outside agencies. This is the essence of research, good practice and clinical audit.

\section{References}

BERNEY, T. P. (1989) 'Consultant Responsibility' paper presented at Section for Psychiatry of Mental Handicap, Banbury.

MACDONALD, N. J. et al (1989) Hypernatraemic dehydration in patients in a large hospital for the mental handicapped. British Medical Journal, 299, 1426-1429. 